\title{
Sentiment and Preference Guided Social Recommendation
}

\author{
Yoke Yie Chen $^{1}$, Xavier Ferrer ${ }^{2,3}$, Nirmalie Wiratunga ${ }^{1}$, and Enric Plaza ${ }^{2}$ \\ ${ }^{1}$ IDEAS Research Institute, Robert Gordon University, Aberdeen, Scotland \\ $\{\mathrm{y} \cdot \mathrm{y} \cdot$ chen, n. wiratunga\}@rgu.ac.uk \\ ${ }^{2}$ Artificial Intelligence Research Institute (IIIA-CSIC) \\ Spanish National Research Council (CSIC) \\ Campus UAB, Bellaterra, Catalonia (Spain) \\ ${ }^{3}$ Universitat Autònoma de Barcelona \\ Bellaterra, Catalonia (Spain) \\ \{xferrer, enric\}@iiia.csic.es
}

\begin{abstract}
Social recommender systems harness knowledge from social experiences, expertise and interactions. In this paper we focus on two such knowledge sources: sentiment-rich user generated reviews; and preferences from purchase summary statistics. We formalise the integration of these knowledge sources by mixing a novel aspect-based sentiment ranking with a preference ranking. We demonstrate the utility of our proposed formalism by conducting a comparative analysis on data extracted from Amazon.com. In particular we show that the performance of the proposed aspect based sentiment analysis algorithm is superior to existing aspect extraction algorithms and that combining this with preference knowledge leads to better recommendations.
\end{abstract}

Keywords: social recommender systems, sentiment analysis, aspect extraction, preference graph

\section{Introduction}

Recommender systems have traditionally relied on improving their ranked lists by exploiting knowledge about user preferences [26], their information needs [11] or by exploiting similar behavior of other users [1]. The huge success of these systems in the retail sector is also its main driving force towards innovative and improved recommendation algorithms. Representation, similarity and ranking algorithms from the Case-Based Reasoning (CBR) community has naturally made a significant contribution to recommender systems research $[18,23]$. The dawn of the social web creates many new opportunities for recommendation algorithms and so the emergence of social recommender systems $[9,12]$.

Consider a typical product recommendation scenario on Amazon (see Figure 1 and Figure 2). Here in addition to typical information about an artefact (e.g. Camera image and textual description), there is also information generated 
or derived from user interactions (e.g. reviews and what users actually buy after viewing this camera). Specifically we observe that there is explicit knowledge in the form of user opinion and implicit knowledge in the form of user preferences. Here by preference we are referring to preference of users over viewed products. Opinion knowledge is often used to improve recommendations by incorporating the sentiment expressed in opinions to bias the retrieved list [9]. Similarly preference knowledge has also separately been applied to successfully influence recommendations [26]. Our focus in this paper is to harness social content by uniting both these knowledge sources to generate better recommendation rankings.

Our contribution is three-fold: firstly we demonstrate how the choice of sentiment analysis algorithms can impact the quality of recommendations; and secondly show how a page-rank type algorithm can be effortlessly incorporated to derive rankings on the basis of preference relationships; and finally provide a formalism to combine sentiment and preference knowledge. Our results confirm that aspect-based sentiment analysis is far superior to one that is agnostic of aspects. This is because purchase choices are based on comparison of artefacts; which implicitly or explicitly involves comparison of characteristics or aspects of these artefacts. Lastly, we introduce a novel algorithm to infer aspect importance weights guided by knowledge represented in a preference graph. Here the insight is that preference relationships modeled in this graph allow us to identify aspects that are likely to have influenced the users' purchase decision. In particular a users purchase decision hints at the aspects that are likely to have influenced their decision and as such be deemed more important.

The rest of the paper is organized as follows: In Section 2 we present the background research related to this work. Next in Section 3 we describe how preference graphs can be generated by using a case study from Amazon.com. The process of aspect extraction and weight learning for sentiment analysis is presented in Section 4. Finally, our evaluation results are presented in Section 5 followed by conclusions in Section 6 .

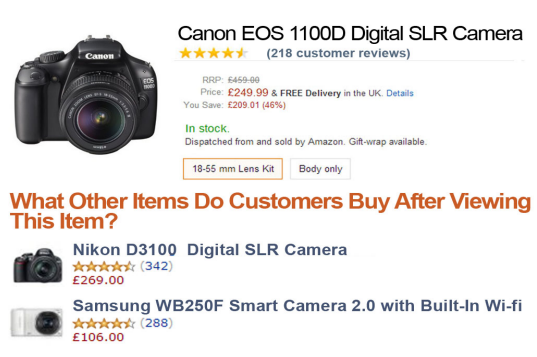

Fig. 1. Product information (explicit).

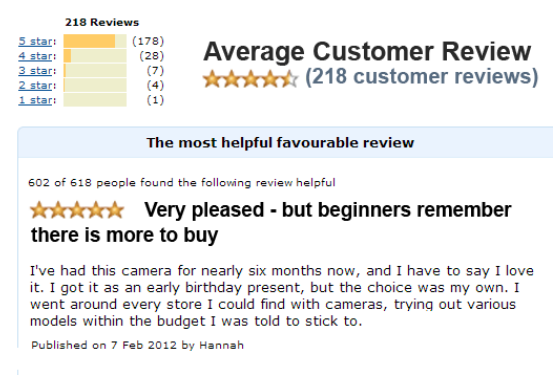

Fig. 2. Product reviews (implicit). 


\section{Related Work}

There exist numerous applications of machine learning and CBR in the area of recommendation systems. Content-based filtering approaches exploit the past and current preferences of the user to build new recommendations. Early work in this area has analysed user logs and sessions [5,17]; reused similar user trails $[2$, 24]; and exploited click-through data [6] to improve recommendations.

Collaborative filtering [15] unlike content-based filtering emphasises the social dimensions of user similarities, preferences and experiences. A good example is Amazon.com, where user ratings and preferences are combined in different ways [16]. In Gupta and Chakraborti [13], graphs are used to model user decisions and combine estimated utilities in the context of preference based recommenders. In Vasudevan and Chakraborti [26], preference models of artefacts are induced based on the trails left by users when critiquing an artefact's aspect. In our work, we do not rely on a preference graph that explicitly defines which aspects are preferred, but instead infer this information from comparing the sentiment-rich content generated by users. Furthermore, unlike other preference based recommender systems which utilise user profile to generate user preferences, we obtained preference knowledge from user interactions. As such we provide a more flexible alternative to preference analysis.

Social recommenders recognise the important role of sentiment analysis of user reviews [9]. Extracting sentiment from natural language constructs is a challenge. Lexicons are often used to ascertain the polarity (positive or negative) and strength of sentiment expressed at word-level (e.g. SentiWordNet [10]). However sophisticated methods are needed to aggregate these scores at the sentence, paragraph and document level to account for negation and other forms of sentiment modifiers [21]. Increasingly aggregation is organised at the aspect level, since the distribution of a user's sentiment is typically mixed and expressed over the aspects of the artefact (e.g. I love the colour but not too keen on size). $\mathrm{Hu}$ and Liu [14] propose an association mining driven approach to identify frequent nouns or noun phrases as aspects. Thereafter sentences are grouped by these aspects and sentiment scores assigned to each aspect group. Whilst there are many other statistical approaches to frequent noun extraction [3,14, 19,22]; others argue that identifying semantic relationships in text provides significant improvements in aspect extraction [20]. Here we explore how semantic based extraction can be augmented by frequency counts.

\section{Social Recommendation Process}

An overview of our proposed process appears in Figure 3. The final outcome is a recommendation of products that are retrieved and ranked on their product rank score, ProdRank, with respect to a given query. Central to this ranking is the integration of sentiment scores derived from user reviews with dominant products inferred from the preference graph.

$$
\operatorname{ProdRank}\left(p_{i}\right)=\alpha * \operatorname{PrefRank}\left(p_{i}\right)+(1-\alpha) * \operatorname{SentiRank}\left(p_{i}\right)
$$




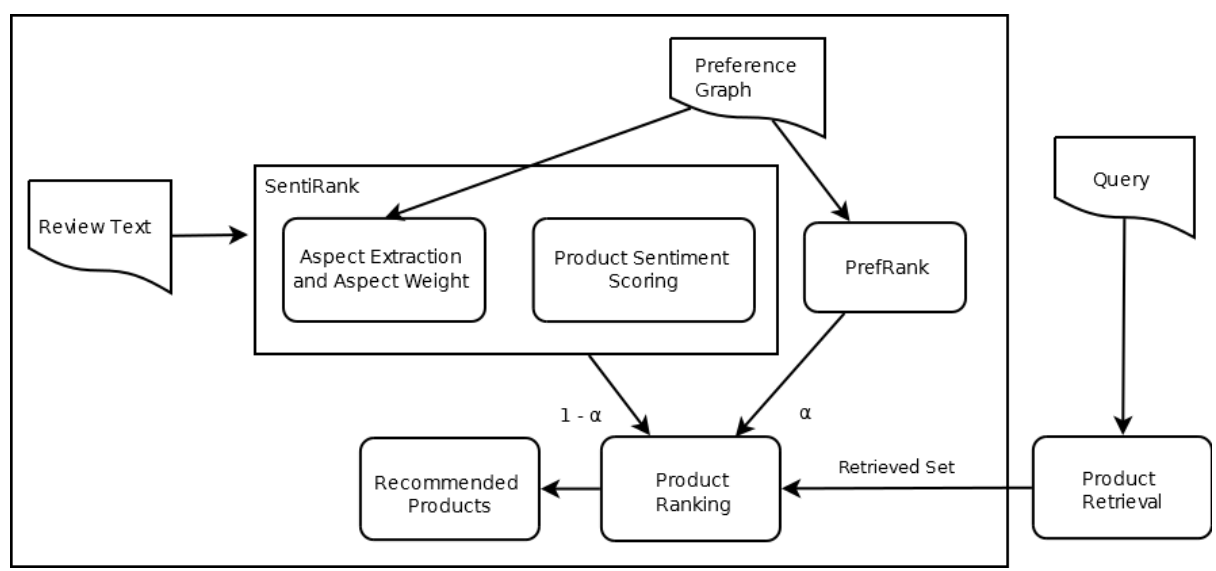

Fig. 3. Overview of social product recommender process.

Where PrefRank assigns a score to product, $p_{i}$, by applying PageRank [4] to a preference graph; and SentiRank assigns a score based on aspect level sentiment analysis. The graph is generated from viewed and purchased product pairs; whilst the sentiment scores are derived from product reviews. We also advocate the use of weighted aspect level sentiment analysis and learn these weights by comparing the sentiment difference between node pairs in the graph (see Section 4). Here $\alpha$ is used as a mixture parameter to study the impact of preference-only ( $\alpha=$ $1)$, sentiment-only $(\alpha=0)$, or a mixture of both $(1<\alpha<0)$.

\subsection{Preference-based Product Ranking}

A preference relation between a pair of products denotes the preference of one product over the other through the analysis of viewed and purchased product relationships. To illustrate the generation of preference relation from Amazon dataset, consider a snapshot of Amazon product web page in Figure 1. Here Canon EOS 1100D Digital SLR Camera (CanonSLR) is the viewed product. At the bottom of this page, we can observed that Nikon D3100 Digital SLR Camera (NikonSLR) and Samsung WB250F Smart Camera (SamsungSmart) are the products that some of the users purchased after viewing CanonSLR. This list of purchased products hints at the preference of users. Using these information, we generate two preference relations in which NikonSLR is preferred over CanonSLR and SamsungSmart is preferred over CanonSLR. A preference graph, $G=(\mathcal{P}, \mathcal{E})$, is generated from such product relation (see Figure 4$)$. The set of nodes, $p_{i} \in \mathcal{P}$, represent products, and the set of directed edges, $\mathcal{E}$, are preference relations, $p_{j} \succ p_{i}$, such that a directed edge from product $p_{i}$ to $p_{j}$ with $i \neq j$ represents that, for some users, $p_{j}$ is preferred over product $p_{i}$. In some cases where $p_{j} \succ p_{i}$ and $p_{i} \succ p_{j}$, a bidrectional preference relation can be observed. For any $p_{i}$, we use $\mathcal{E}^{i}$ to denote in-coming and $\mathcal{E}_{i}$ for outgoing product sets. 


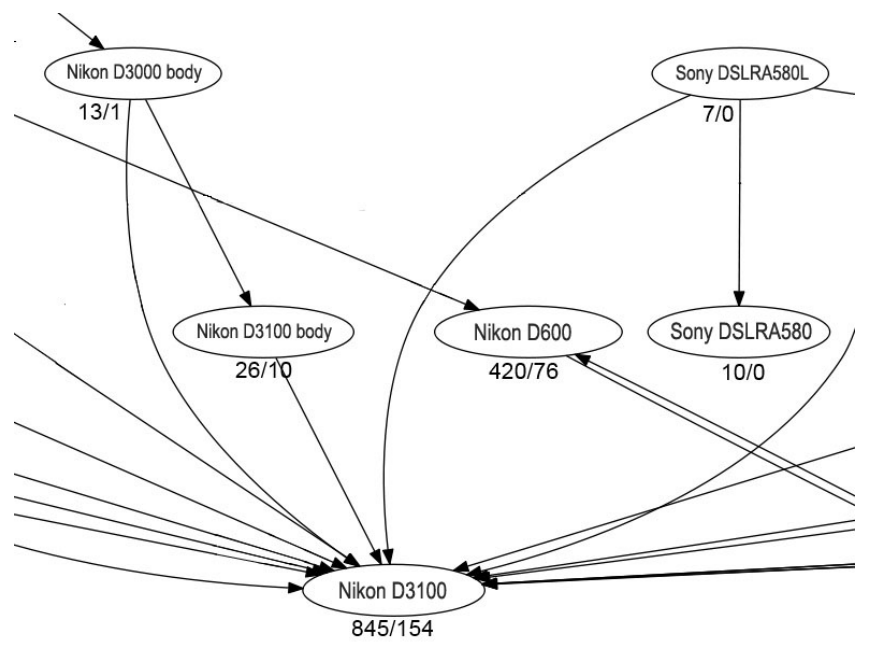

Fig. 4. Preference sub-graph for Amazon Digital SLR Cameras.

Figure 4 illustrates a preference graph generated from a sample of Amazon data on Digital SLR Camera. The number of reviews/questions for a product is shown below each product node. Typically top ranked products have many more incoming edges, while less popular products tend to have more outgoing edges. It is not surprising that product Nikon D3100 in Figure 4 is listed in Amazon's top 10 Best Seller ranking. Based on these observations, the higher the number of incoming edges (quantity) from preferred products (quality), the higher the preference rank, PrefRank, is for product, $p_{i}$.

$$
\operatorname{PrefRank}\left(p_{i}\right)=\sum_{p_{j} \in \mathcal{E}^{i}} \frac{\operatorname{PrefRank}\left(p_{j}\right)}{\left|\mathcal{E}_{j}\right|}
$$

Where $\mathcal{E}^{i}$ is the set of all viewed products over which $p_{i}$ is preferred and $\mathcal{E}_{j}$ is the set of products that are preferred after viewing $p_{i}$.

The main assumption here is similar to PageRank where the score captures the importance of pages by the analysis of the quantity and quality of edges to pages [4]. However we also observed that while our assumption is true with most studied products, it is not always the case that a product with higher PrefRank will also have a higher rank in Amazon's Best Seller. For example, Amazon's third ranked camera has a higher PrefRank score compared to the second ranked camera (see Table 1). Similar observations also hold when comparing PrefRank scores with user generated overall product ratings. This motivates the need to leverage on further dimensions of knowledge sources such as sentiment from product reviews for product recommendation. 


\begin{tabular}{|l||l|l|l|l||l|l|l||}
\hline \multirow{2}{*}{$\begin{array}{l}\text { Amazon } \\
\text { Bestseller } \\
\text { Ranking }\end{array}$} & \multicolumn{3}{|c|}{ PrEFERENCE } & \multicolumn{3}{|c|}{ SENTIMENT } \\
\cline { 2 - 7 } & PrefRank & $\begin{array}{l}\text { Incoming } \\
\text { Edges }\end{array}$ & $\begin{array}{l}\text { Outgoing } \\
\text { edges }\end{array}$ & $\begin{array}{l}\text { \#Reviews } \\
\text { per Day }\end{array}$ & $\begin{array}{l}\text { SentiRank } \\
\text { aspects }\end{array}$ & \#Reviews & $\begin{array}{l}\text { Product } \\
\text { Rating }\end{array}$ \\
2 & 0.1240 & 26.0 & 1.0 & 1.136 & 0.087 & 1351.0 & 4.7 \\
3 & 0.0037 & 2.0 & 2.0 & 0.770 & 0.106 & 242.0 & 4.7 \\
$4-5$ & 0.0178 & 10.0 & 0.0 & 0.621 & 0.097 & 847.0 & 4.6 \\
$6-10$ & 0.0053 & 1.5 & 0.5 & 0.589 & 0.102 & 129.0 & 4.8 \\
$11-50$ & 0.0435 & 5.5 & 1.25 & 0.453 & 0.089 & 354.75 & 4.72 \\
$51-100$ & 0.0048 & 2.071 & 0.892 & 0.506 & 0.080 & 407.35 & 4.45 \\
N/A & 0.0021 & 0.529 & 1.647 & 0.366 & 0.124 & 393.64 & 4.52 \\
\end{tabular}

Table 1. Summary of the averages of products organised by Amazon Best Seller rank.

\section{Aspect Weighted Sentiment-based Product Ranking}

Users author reviews following the purchase of products. These contain user opinion in the form of positive and negative sentiment. Strength of sentiment expresses the intensity with which an opinion is stated with reference to a product [25]. We exploit this information as a means to rank our products, such that products ranked higher denote higher positive sentiment. SentiRank of a product, $p_{i}$, given a set of related reviews $\mathcal{R}^{i}$ is computed as follows:

$$
\operatorname{SentiRank}\left(p_{i}\right)=\frac{\sum_{k=1}^{\left|\mathcal{R}^{i}\right|} \operatorname{SentiScore}\left(r_{k}\right)}{\left|\mathcal{R}^{i}\right|}
$$

Here SentiScores are generated by the SMARTSA system [21] for each $r_{k} \in \mathcal{R}^{i}$. The SMARTSA system obtains the sentiment score of sentiment-bearing words from SentiWordNet [10]. The score will be modified to take into consideration of negation terms and lexical valence shifters (e.g. intensifier and diminish terms). The negative and positive strength is expressed as a value in the range [-1:1].

A finer-grained analysis of reviews is achieved by computing sentiment at the aspect level. It allows the sentiment of product, $p_{i}$, to be associated with individual aspects $a_{j} \in \mathcal{A}^{i}$ where $\mathcal{A}^{i} \subseteq \mathcal{A}$. The aspects of a product are extracted by using Algorithm 1. Accordingly we have the following rewrite for SentiRank.

$$
\operatorname{SentiRank}\left(p_{i}, a_{j}\right)=\frac{\sum_{j=1}^{\left|\mathcal{A}^{i}\right|} \text { AspectWeight }\left(a_{j}\right) * \text { AspectSentiScore }\left(p_{i}, a_{j}\right)}{\left|\mathcal{A}^{i}\right|}
$$

This new formalisation is a weighted summation of sentiment expressed at the aspect level. Once aspects are extracted the sentiment of a product's reviews can be expressed as a distribution over each aspect. Accordingly, the aspectlevel sentiment score is:

$$
\operatorname{AspectSentiScore}\left(p_{i}, a_{j}\right)=\frac{\sum_{m=1}^{\left|\mathcal{R}_{j}^{i}\right|} \operatorname{SentiScore}\left(r_{m}\right)}{\left|\mathcal{R}_{j}^{i}\right|} *(1-\text { Gini })
$$


Where $\mathcal{R}_{j}^{i}$ is a set of reviews for product $p_{i}$ related to aspect $a_{j}$ and $r_{m} \in \mathcal{R}_{j}^{i}$. We use the Gini coefficient [27] to assign higher sentiment scores to an aspect when there is consensus about the distribution of the sentiment and otherwise is penalised accordingly.

A product purchase choice is a preference made on the basis of one or more aspects. The notion of aspect importance arises when the same set of aspects contribute to similar purchase decisions. Using this same principle, aspects weights are derived by comparing the aspect sentiment score differences between purchased and viewed product pairs in which $\left(p_{x}, p_{y}\right) \in\left\{\left(p_{x}, p_{y}\right)\right\}_{x, y=1 \wedge x \neq y}^{t}$

$$
\text { AspectWeight }\left(a_{j}\right)=\frac{\sum_{x=1}^{|\mathcal{P}|} \sum_{y=1}^{|\mathcal{P}|} \delta\left(a_{j}, p_{x}, p_{y}\right)}{|t \in \mathcal{E}|}
$$

where either $p_{x} \succ p_{y}$ or $p_{y} \succ p_{x}$ or both, and $t$ is the set of product preference pairs containing aspect $a_{j}$. The preference difference between any pairs of products is computed as:

$$
\begin{gathered}
\delta\left(a_{j}, p_{x}, p_{y}\right)=\left|L_{\min }(\mathcal{A}, \mathcal{E})\right|+\delta^{\prime}\left(a_{j}, p_{x}, p_{y}\right) \\
\delta^{\prime}\left(a_{j}, p_{x}, p_{y}\right)=\left(\text { AspectSentiScore }\left(a_{j}, p_{x}\right)-\text { AspectSentiScore }\left(a_{j}, p_{y}\right)\right)
\end{gathered}
$$

Here $\left|L_{\min }(\mathcal{A}, \mathcal{E})\right|$ is the lowest preference difference scores obtained over all the aspect and product preference pairs. This is required to avoid negative aspect weights while preserving the importance of the aspects.

\subsection{Sentiment Aspect Extraction}

Grammatical extraction rules [20] are used to identify a set of candidate aspect phrases from sentences. These rules operate on dependency relations in parsed sentences $^{1}$. Figure 5 lists the rules that we have employed in this work. Here $N$ is a noun, $A$ an adjective, $V$ a verb, $h$ a head term, $m$ a modifier and $\langle h, m\rangle$ is a candidate phrase. Examples of how these rules apply to sample sentences appear in Table 2. Consider the first example sentence which according to Algorithm 1 applies to rule three: $\operatorname{cop}($ good, is $)+n s u b j($ good,lens $) \rightarrow\langle$ lens, good $\rangle$. Next, if a Noun Compound Modifier $(n n)$ exists in the sentence, rules five and six apply; and in this example rule five applies resulting in the following candidate aspects: $($ lens, good $)+n n($ lens, camera $) \rightarrow\langle$ camera lens, good $\rangle$. In this way given a set of reviews a set of candidate phrases are extracted. For each candidate, non noun $(N)$ words are eliminated. Thereafter frequency of each candidate is calculated according to its $N$ and $N N$ phrase; retaining only those candidates above a frequency cut-off (e.g. greater than $1 \%$ of the maximum frequency occurrence of a noun).

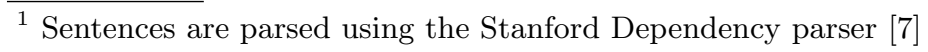




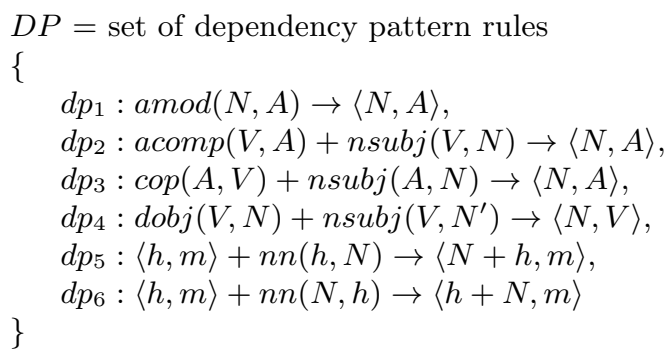

Fig. 5. Extraction rules.

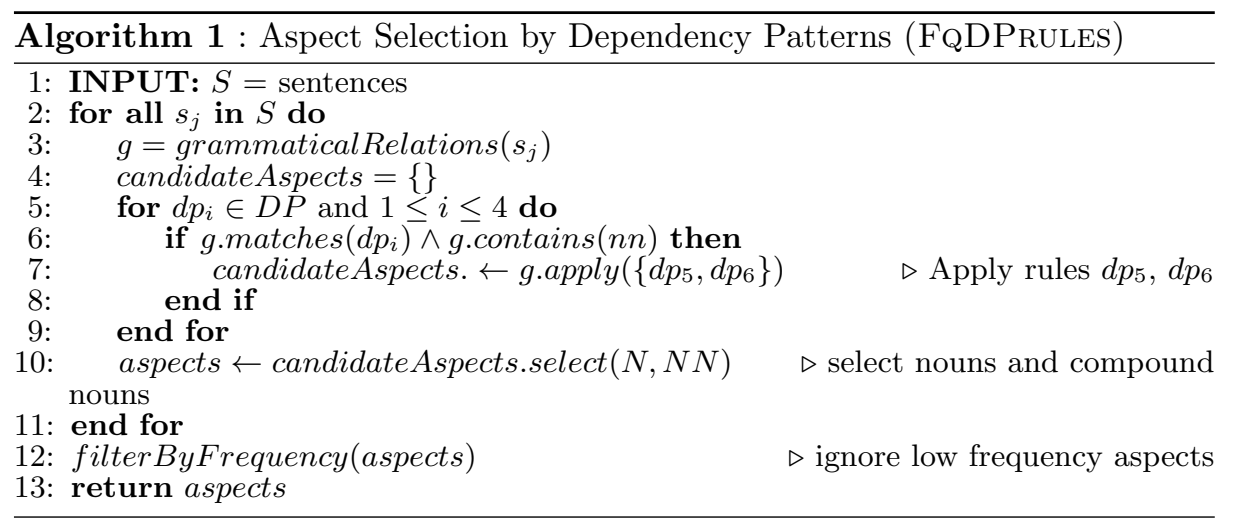

\subsection{Aspect Weight Extraction}

Learning of aspect weights relies on preference graph and aspect level sentiment knowledge. Figure 6 and 7 illustrates the notion of preference difference calculations using a trivial three node preference graph. In Figure 6, the relation $p_{3}(+0.1) \succ p_{1}(+0.8)$ denotes product $p_{3}$ is preferred over $p_{1}$ and they have an aspect sentiment score of +0.1 and +0.8 respectively for aspect lens (see Equation 5). Corresponding preference difference scores are also shown in Table 3 for two aspects. Here lens and screen have a normalised aspect weights of 0.03 and 0.97 respectively. Therefore, we suggest that aspect screen is more important than aspect lens.

\section{Evaluation}

In this section we evaluate our proposed integration of sentiment and preference knowledge applied to product recommendation. Since the quality of extracted aspects in sentiment analysis will have a direct impact on the quality of recommendations; we first conduct a pilot study to evaluate the quality of aspects extracted by our algorithm with the state-of-the-art. Thereafter, we evaluate 


\begin{tabular}{|l|l|l|}
\hline Example & Grammatical relations & Dependencies \\
\hline The camera lens is good & $\begin{array}{l}\text { nsubj (Nominal Subject) } \\
\text { cop (Copula) } \\
\text { nn (Noun Compound Modifier) }\end{array}$ & $\begin{array}{l}\text { nsubj(good, lens) } \\
\text { cop(good, is) } \\
\text { nn(lens, camera) }\end{array}$ \\
\hline The screen is bright with nice colors & amod (Adjectival Modifier) & amod(colors, nice) \\
\hline She looks amazing & acomp (Adjectival Complement) & acomp(looks, amazing) \\
\hline I like the camera lens & dobj (Direct Object) & dobj(like, lens) \\
\hline
\end{tabular}

Table 2. Definition of grammatical relations.

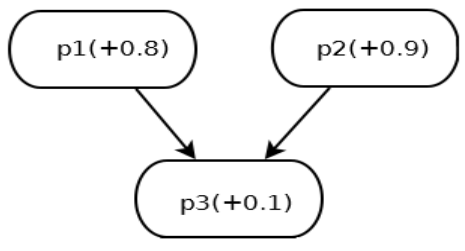

Fig. 6. Sub-graph lens aspect.

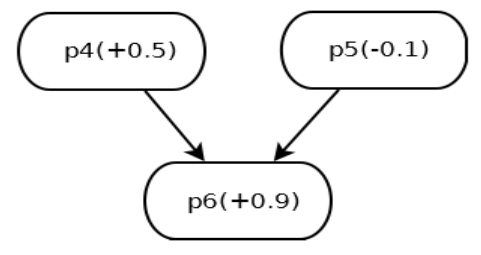

Fig. 7. Sub-graph screen aspect.

how well the recommendation system works in practice on Amazon.com data using two derived benchmark rankings. Here we are keen to study the complimentary roles of sentiment and preference knowledge and expect to discover a synergistic relation between them.

\subsection{Comparative Pilot Study - Aspect Extraction Analysis}

We use a public dataset on product reviews containing manually marked-up product aspects $[8,14]$. For this study we use phone category products with at least hundred reviews. Precision and recall is used to compare manually labeled aspects with extracted ones using the following alternative extraction algorithms:

- FQITEMS uses shallow NLP to identify single nouns as candidate aspects that are then pruned using a frequency cut-off threshold.

- FQPos uses Part-of-Speech(POS) extraction patterns that are then pruned using sentiment informed frequency cut-off threshold [9]

- DPRules uses the dependency extraction rules in Figure 5 [20].

- FQDPRUles same as DPRULES but prunes candidate aspects using a frequency cut-off (See Algorithm 1).

Precision of all frequency based extraction approaches are significantly better compared to DPRUles (see Table 4). We also confirm that FQPos improves over FQITEMS. As expected best results are achieved with FQDPRULES when deep NLP semantics is combined with frequency prunning. Here we observe a $26 \%$ and 7\% improvement in precision over FQITEMS and FQPOS respectively. Recall trends suggests that FQDPRULES must have many false negatives and so missed extraction opportunities compared to FQITEMS and FQPOS. However a lower precision is more damaging as it is likely to introduce aspect sparsity problems 


\begin{tabular}{|c|cc|c|}
\hline Aspects & $\begin{array}{c}\text { Preference } \\
\text { Relations }\end{array}$ & $\begin{array}{c}\text { Preference } \\
\text { Difference } \\
\text { Scores }\end{array}$ & $\begin{array}{c}\text { Normalised Aspect } \\
\text { Weights }\end{array}$ \\
\hline \multirow{2}{*}{ lens } & $p_{3} \succ p_{1}$ & $=0.1$ & $\frac{0.1}{3.1}=0.03$ \\
& $p_{3} \succ p_{2}$ & $=0.0$ & $\frac{3.0}{3.1}=0.97$ \\
\hline \multirow{2}{*}{ screen } & $p_{6} \succ p_{4}$ & $=1.2$ & $=1.8$ \\
& $p_{6} \succ p_{5}$ & $=1.8$ & \\
\hline
\end{tabular}

Table 3. Aspect preference scores.

\begin{tabular}{|l||c|c|c|}
\hline Approach & Precision & Recall & F-score \\
\hline FQITEMS & 0.60 & 0.34 & 0.43 \\
FQPoS & 0.71 & 0.11 & 0.20 \\
DPRULES & 0.28 & 0.66 & 0.39 \\
FQDPRULES & 0.76 & 0.25 & 0.37 \\
\hline
\end{tabular}

Table 4. Results for aspect extraction pilot study.

which have detrimental effect on sentiment difference computations. Therefore on the basis of these results we use FQDPRULES to extract product aspects for sentiment analysis in the social recommender experiments in the next sections.

\subsection{Amazon Dataset}

We crawled 2264 Amazon products during April 2014. From this we use the Digital SLR Camera category containing more than 20,000 user generated reviews. Since we are not focusing on the cold-start problem, newer products and those without many user reviews are removed. Here we use 1st January 2008 and less than 15 reviews as the prunning factor for products. Finally, any synonymous products are united leaving us data for 80 products (see Table 5).

The FQDPRULES algorithm extracted 981 unique aspects and on average 128 different aspects for each product. Importantly more than $50 \%$ of the products shared at least 70 different aspects, while $30 \%$ shared more than 90 aspects on average. The fact that there are many shared aspects is reassuring for product comparison.

\begin{tabular}{|ll|}
\hline \#Products & 2,264 \\
\#Products (filtered) & 80 \\
\#Reviews (filtered) & 21,034 \\
\#Aspects Mean (Std. Dev.) & 128.66 (43.84) \\
\#Different aspects & 981 \\
\hline
\end{tabular}

Table 5. Amazon Digital SLR Camera dataset.

\subsection{Ranking Strategies}

The retrieval set of a query product consists of products that share a similar number of aspects. This retrieval set is ranked using the following sentiment- 
based recommendation strategies in which the formalisations were presented in Section 4:

- BASE: recommend using sentiment in reviews (see Equation 3);

- AspectG: recommend using aspect sentiment analysis (see Equation 5);

- Aspect: same as AsPECTG but without the Gini coefficient weighting in Equation 5; and

- AspectG*: same as AspectG but with the additional aspect weighting component in Equation 4.

We also present each of the above strategies in combination with preference knowledge (see Equation 2). Finally, the impact of increasing $\alpha$ values is analysed according to Equation 1 to study the relationship between sentiment and preference knowledge.

\subsection{Evaluation Metrics}

In the absence of a manual qualitative estimate of recommendation or access to user specific purchase trails, we derived approximations from the Amazon data we had crawled. For this purpose, using a leave-one-out methodology, the average gain in rank position of recommended products over the left-out query product is computed relative to a benchmark product ranking.

$$
\% \text { RankGain }=\frac{\sum_{i=1}^{n=3} \text { benchmark }\left(P_{q}\right)-\operatorname{benchmark}\left(P_{i}\right)}{n *|\mathcal{P}-1|}
$$

where $n$ is the size of the retrieved set and benchmark returns the position on the benchmark list. The greater the gain over the query product the better. Suppose the query product is ranked 40th on the benchmark list of 81 unique products $\mathcal{P}$, and the recommended product is ranked 20th on this list, then the recommended product will have a relative benchmark rank improvement of $25 \%$.

We generate two benchmark lists according to the following dimensions:

- Star-Rating: Use the Amazon star rating. However, this benchmark is static and tends to be less reliable as it does not necessarily represent the current trends about products. For example, the LCD display on a camera may have been a good reason for a high star rating in the past whilst now almost every camera possesses it.

- Popular: Derived from Amazon's reviews, questions and timeline data.

$$
\operatorname{Popular}(p)=\frac{\text { nReviews }+n Q u e s t i o n s}{\text { days_online }}
$$

where nReviews and nQuestions refer to the number of reviews and questions of a product respectively, and days_online is the number of days the product has been on Amazon's website. We found that this formula has some 
correlation with the top 100 Amazon Best Seller ranking (Spearman correlation of -0.4381). Unlike Amazon's benchmark this allows us to experiment with query products that may not necessarily be in the Top $100^{2}$.

\subsection{Results}

The graphs in Figures 8 and 9 show for increasing numbers of shared aspects the performance of each algorithm in terms of the average \%RankGain on benchmarks Popular and Star-Rating. In general AsPeCT and AsPECTG perform best, with both strategies recommending products with an average rank gain of close to $15 \%$ compared to BASE with no aspect extraction. It is worth pointing out that the improvement with applying the Gini coefficient is approximately $5 \%$ more on average when compared to not using it.

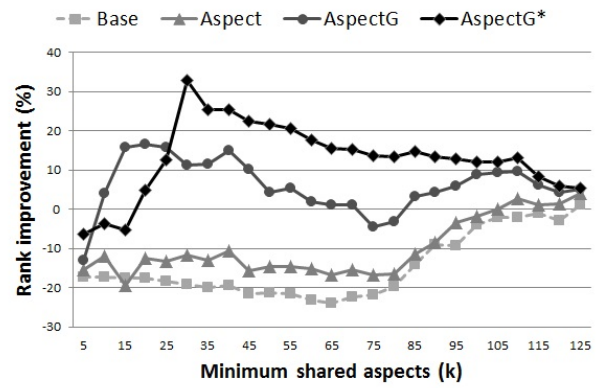

Fig. 8. Benchmark Popular.

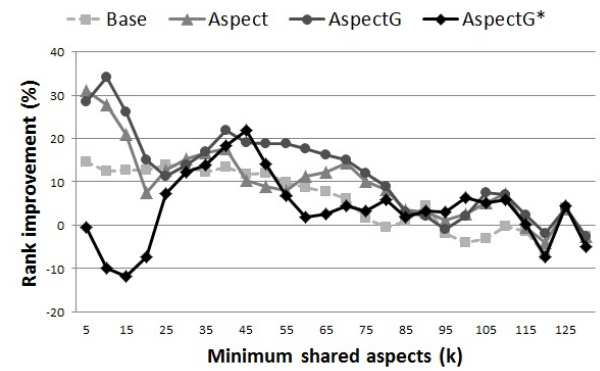

Fig. 9. Benchmark Star-Rating.

Results when using aspect weights with AsPECTG* is mixed and seems dependent on the benchmark used. For instance it does very poorly on the static Star-Rating benchmark yet is the winning algorithm on the Popular benchmark. In fact it achieves up to $30 \%$ gain (with 30 shared aspects or more with the query product) on the Popular benchmark. One explanation for its poor performance on Star-Rating might be explained by the fact that user preferences about camera aspects may have changed during the period of 2008-2014. Since aspect weights are learnt on the basis of preference knowledge it may well be that similar information is implicitly influencing the Popular benchmark.

Table 6 shows the top 5 most and less preferred aspects together with their weights and frequency of appearance in products. Note that the sum of aspects weights is 1 . Here the shutter aspect seems to be very important for Amazon users whilst deal, found in 67 different products, is about 30 times less appreciated. It is interesting to note that aspect frequencies are equally distributed, ranging from 59 to 70 in our set formed by 80 products, however the weight distribution provides a finer-grained differentiation.

\footnotetext{
$\overline{2}$ http://www. amazon.co.uk/Best-Sellers-Electronics-Digital-SLR-Cameras
} 


\begin{tabular}{ccc||ccc}
\hline \multirow{2}{*}{ Top 5 Popular Aspects } & \multicolumn{3}{c}{ Bottom 5 Popular Aspects } \\
Aspect & $\begin{array}{c}\text { Weight } \\
\text { (normalised) }\end{array}$ & $\begin{array}{c}\text { Frequency } \\
\text { (No. of } \\
\text { products) }\end{array}$ & Aspect & $\begin{array}{c}\text { Weight } \\
\text { (normalised) }\end{array}$ & $\begin{array}{c}\text { Frequency } \\
\text { (No. of } \\
\text { products) }\end{array}$ \\
\hline $\begin{array}{c}\text { shutter } \\
\text { photography }\end{array}$ & 0.00229 & 61 & deal & 0 & 67 \\
point & 0.00181 & 70 & lcd & $8.56 \cdot 10^{-5}$ & 59 \\
system & 0.00179 & 70 & control & $2.06 \cdot 10^{-4}$ & 66 \\
video & 0.00176 & 54 & day & $2.92 \cdot 10^{-4}$ & 70 \\
\hline
\end{tabular}

Table 6. Preferred aspects for Digital SLR Cameras in Amazon.

Next we study the mixing parameter $\alpha$ using 90 shared aspects in Figure 10 and Figure 11; where high values of $\alpha$ mean PrefRank having a greater influence on ProdRank compared to that of SentiRank. In general we observe that increasing values of $\alpha$ lead to increasing gain in benchmark positions. This is most notable on the Popular benchmark, where it achieves a maximum of $24 \%$ with all four strategies; whilst showing only a very modest gain of $3 \%$ with $\alpha=0.9$ on the Star-Rating benchmark.

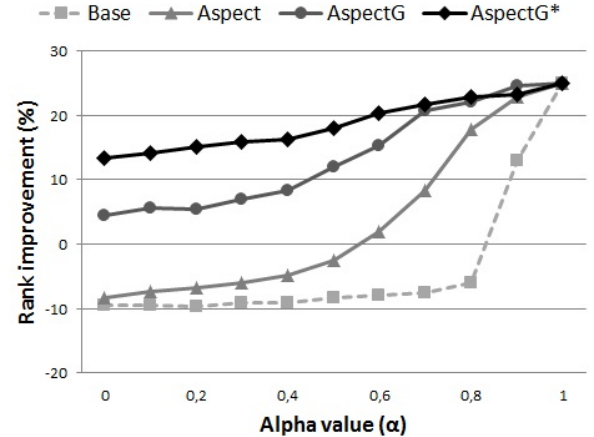

Fig. 10. ProdRank on Popular.

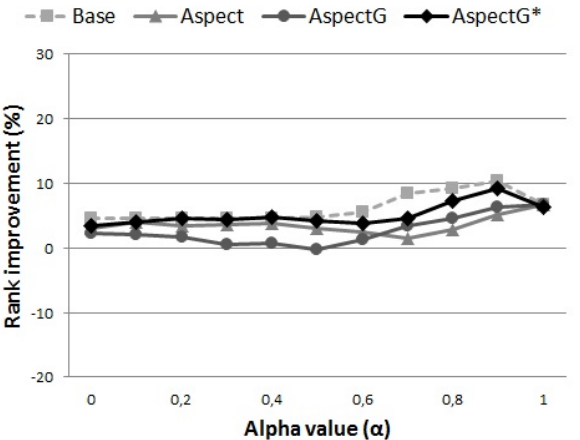

Fig. 11. ProdRank on Star-Rating.

\section{Conclusion}

Social recommenders have created new opportunities for CBR research based on harnessing and reusing people's online experiences. The volume and variety of social media calls for innovative extraction, representation, retrieval and knowledge integration algorithms.

In this paper we formalised the extraction and integration of two social media sources: sentiment knowledge in product reviews; and preferences from purchase 
summary statistics. The benefits are demonstrated in a realistic recommendation setting using benchmarks generated from social media. We show that higher precision in aspect extraction was achieved when grammatical rules are combined with support statistics. Importantly there was over $50 \%$ shared aspects on average between any product pair, providing for non-sparse aspect-level representations. We confirm that preference knowledge can be conveniently exploited using the PageRank algorithm, and demonstrated the benefits of inferring aspect weights from preference graphs.

In the absence of ground truth data, generation of benchmark data becomes important for recommender research in general. Whilst there are a variety of social data dimensions that can be used to derive benchmark metrics, further work is needed to understand the interactions between these dimensions. Our results show that the combination of sentiment and preference knowledge are promising, but further work is needed to study closely the role of each on different domains and benchmarks. Finally it would also be interesting to explore how aspect importance weights are likely to evolve with context and time.

Acknowledgments. This research has been partially supported by AGAUR Scholarship (2013FI-B 00034) and Project Cognitio TIN2012-38450-C03-03.

\section{References}

1. G. Adomavicius and A. Tuzhilin. Toward the next generation of recommender systems: A survey of the state-of-the-art and possible extensions. Knowledge and Data Engineering, IEEE Transactions on, 17, 2005.

2. D. Billsus and M. Pazzani. A hybrid user model for news story classification. Courses and lectures - Inter. Center for mechanical sciences, 99, 1999.

3. S. Blair-Goldensohn, K. Hannan, R. McDonald, T. Neylon, G. Reis, and J. Reynar. Building a sentiment summarizer for local service reviews. In $W W W$ Workshop on NLP in the Information Explosion Era, page 14, 2008.

4. S. Brin and L. Page. The anatomy of a large-scale hypertextual web search engine. Computer networks and ISDN systems, 30(1):107-117, 1998.

5. R. Burke. Hybrid recommender systems: Survey and experiments. User Modeling and User-Adapted Interaction, pages 331-370, 2002.

6. B. Cao, D. Shen, K. Wang, and Q. Yang. Clickthrough log analysis by collaborative ranking. In AAAI Conf. on Artificial Intelligence, 2010.

7. M. De Marneffe, B. MacCartney, C. Manning, et al. Generating typed dependency parses from phrase structure parses. In Proc. of Language Resources and Evaluation Conference, pages 449-454, 2006.

8. Xiaowen Ding, Bing Liu, and Philip S. Yu. A holistic lexicon-based approach to opinion mining. In Proc. Int. Conf. on Web Search and Data Mining, 2008.

9. R. Dong, M. Schaal, M. OMahony, K. McCarthy, and B. Smyth. Opinionated product recommendation. In Inter. Conf. on Case-Based Reasoning, 2013.

10. A. Esuli and F. Sebastiani. Sentiwordnet: A publicly available lexical resource for opinion mining. In Proc. Language Resources and Evaluation Conference, pages 417-422, 2006. 
11. S. Gauch, M. Speretta, A. Chandramouli, and A. Micarelli. User profiles for personalized information access. In The adaptive web, pages 54-89. Springer-Verlag, 2007.

12. G. Groh, S. Birnkammerer, and V. Köllhofer. Social recommender systems. In Recommender Systems for the Social Web, pages 3-42. Springer Berlin Heidelberg, 2012.

13. S. Gupta and S. Chakraborti. Utilsim: Iteratively helping users discover their preferences. In E-Commerce and Web Technologies, pages 113-124, 2013.

14. M. Hu and B. Liu. Mining and summarising customer reviews. In Proc. of ACM SIGKDD Inter. Conf. on Knowledge Discovery and Data Mining, KDD '04, pages 168-177, 2004.

15. S. Kim, P. Pantel, T. Chklovski, and M. Pennacchiotti. Automatically assessing review helpfulness. In Proc. Conf. on Empirical Methods in Natural Language Processing, pages 423-430, 2006.

16. G. Linden, B. Smith, and J. York. Amazon. com recommendations: Item-to-item collaborative filtering. Internet Computing, IEEE, 7(1):76-80, 2003.

17. K. McCarthy, Y. Salem, and B. Smyth. Experience-based critiquing: reusing critiquing experiences to improve conversational recommendation. In Inter. Conf. on Case-Based Reasoning, pages 480-494, 2010.

18. L. McGinty and B. Smyth. Collaborative case-based reasoning: Applications in personalised route planning. In Case-Based Reasoning Research and Development, pages $362-376,2001$.

19. S. Moghaddam and M. Ester. Opinion digger: An unsupervised opinion miner from unstructured product reviews. In Proc. Inter. Conf. on Information and Knowledge Management, CIKM '10, 2010.

20. S. Moghaddam and M. Ester. On the design of lda models for aspect-based opinion mining. In Proc. Inter. Conf. on Information and Knowledge Management, CIKM '12, 2012.

21. A. Muhammad, N. Wiratunga, R. Lothian, and R. Glassey. Contextual sentiment analysis in social media using high-coverage lexicon. In Research and Development in Intelligent Systems, pages 79-93, 2013.

22. A. Popescu and O. Etzioni. Extracting product features and opinions from reviews. In Natural language processing and text mining, pages 9-28. Springer London, 2007.

23. L. Quijano-Sánchez, D. Bridge, B. Díaz-Agudo, and J. Recio-García. Case-based aggregation of preferences for group recommenders. In Case-Based Reasoning Research and Development, pages 327-341, 2012.

24. A. Singla, R. White, and J. Huang. Studying trailfinding algorithms for enhanced web search. In Proc. ACM SIGIR Conf. on Information Retrieval, pages 443-450, 2010.

25. P. Turney. Thumbs up or thumbs down?: semantic orientation applied to unsupervised classification of reviews. In Proc. Annual Meeting on Association for Computational Linguistics, pages 417-424, 2002.

26. S. Vasudevan and S. Chakraborti. Mining user trails in critiquing based recommenders. In Proc. Inter. Conf. on World Wide Web Companion, pages 777-780, 2014.

27. S. Yitzhaki. Relative deprivation and the Gini coefficient. The Quarterly Journal of Economics, pages 321-324, 1979. 